\title{
RETRACTED ARTICLE: Surveillance of Suspicious Activities of Chinese Army Near Indian LAC
}

\author{
Aditya Kakde $^{1} \cdot$ Bhavana Kaushik $^{1} \cdot$ Durgansh Sharma $^{2} \cdot$ Neelu Jyoti Ahuja $^{1}$
}

Received: 6 November 2020 / Accepted: 10 November 2020/ Published online: 24 November 2020

(C) Indian Society of Remote Sensing 2022

The Editor-in-Chief has retracted this article because it was published in error before the peer review process was completed. The content of this article has been removed for legal reasons. The authors have been offered to submit a revised manuscript for further peer review. All authors agree with this retraction.

Aditya Kakde

adityakakde100@gmail.com

1 Department of Systemic, UPES, Dehradun, India

2 Department of Cybernetics, UPES, Dehradun, India 